\title{
Sets of Piezoelectric Probeheads for Stress Evaluation with Subsurface Waves
}

\author{
Jacek Szelążek
}

Received: 14 August 2012 / Accepted: 25 January 2013 / Published online: 9 March 2013

(C) The Author(s) 2013. This article is published with open access at Springerlink.com

\begin{abstract}
This paper discusses the development of piezoelectric ultrasonic probeheads for measurements of time of flight of subsurface or surface wave ultrasonic pulses. Sets of such probes found application in ultrasonic stress evaluation. In precise measurements of time of flight using piezoelectric transducers, the main source of time scatter proved to be variation in the condition of liquid couplant layers. The thickness of this couplant layer depends on surface roughness of the sample being evaluated. Paper describes a new configuration of probes, composed of two transmitting and four receiving probes arranged along one line, designed to reduce coupling influence on readings. Presented is comparison of readings obtained with known sets of probes and with a new one. Also presented are results of residual stress evaluation performed with various 6-transducer sets of probes on railroad rails, monoblock railroad wheels and pillars of hydraulic press. All measurements were performed in industrial conditions, without any special surface preparation.
\end{abstract}

Keywords Ultrasonic testing - Piezoelectric probes · Evaluation of surface stress

\section{Introduction}

One of the relatively novel applications of ultrasonic technique is the determination of stresses in various structures

\section{J. Szelążek $(\bowtie)$}

Institute of Fundamental Technological Research, Polish Academy of Sciences, Pawińskiego Str. 5B, 02-106 Warsaw, Poland

e-mail: jszela@ippt.gov.pl or elements. Stress evaluation based on the acoustoelastic effect concerns ultrasonic wave velocity changes due to stress. Theoretical relationships for velocity-stress were presented in 1953 by Hughes and Kelly [1] and shortly later demonstrated experimentally. The first demonstration of acoustoelastic effects for stress measurements were presented by Bergman and Shahbender 1958 [2], and a few years later the first industrial experiment to evaluate hoop residual stress in railroad monoblock wheels was described [3].

In numerous experiments it was shown that in the elastic regime, a linear dependence of velocity as a function of stress is observed. For example, [4] describes such measurements in steel wires, [5] in cast steel, [6] in forged steel and [7] presents results of measurements in wood. Also, the dependence of stress induced velocity changes on material structure was investigated [8].

For an ultrasonic wave propagating in a solid along the stress direction, the wave velocity or time of flight (TOF) of ultrasonic pulse changes due to stress are described as:

$\sigma_{i} \beta_{i j k}=\frac{\left(V_{i}-V_{i}^{0}\right)}{V_{i}^{0}}=\frac{\left(t^{0}-t\right)}{t}$

where $\sigma_{i}$-stress, $\beta$-acoustoelastic constant, indexes $i, j$, $k$ denotes directions of propagation, polarization and stress respectively, $V_{i}^{0}, V_{i}$ and $t^{0}, t$-velocities and times of flight in stress-free and stressed conditions, respectively.

Equation (1) allows one to calculate a stress value assuming the acoustoelastic constant $\beta$ is known and velocities or TOF are measured in stress-free and stressed conditions. If values of second and third order elastic constants for a material under test are known, the value of the acoustoelastic constant can be also calculated according to formulas presented in [1]. Most often, however, acoustoelastic constants are determined experimentally on samples during tensile testing. 
Unfortunately, in industrial metals such as steels or light alloys, stress induced velocity changes are small. For example, the most stress sensitive wave (exhibiting the highest value of acoustoelastic constant), a longitudinal wave propagating parallel to the stress direction in steel for a distance of $100 \mathrm{~mm}$, under an applied $10 \mathrm{MPa}$ tensile stress, results in an increase in TOF equal to only $1.35 \mathrm{~ns}$ (calculated for longitudinal wave velocity in steel of $5.94 \mathrm{~m} / \mathrm{s}$ and an acoustoelastic constant $\beta_{111}=-1.25 \cdot 10^{-5} \mathrm{MPa}^{-1}$ ). This means that to determine the value of stress with sufficient precision it is necessary to measure TOF with nanosecond accuracy. Modern electronics allow the measurement of TOF with high, picosecond accuracy, in laboratory conditions and on specially prepared samples. Greater difficulty is encountered in performing precise and repeatable measurements of TOF in industrial conditions, on components and structures, in a nondestructive way without any surface machining. The aim of the paper is to present set of piezoelectric probes used to measure the TOF of subsurface waves, on elements exhibiting rough surfaces, in industrial conditions.

\section{Methods of Ultrasonic Wave Velocity Measurements}

\subsection{Pulse-Echo Method}

There are, in general, two types of ultrasonic wave velocity or TOF measurements. The first is a pulse-echo method, useful in measurements on elements with two parallel surfaces, such as plates. TOF is measured between consecutive echoes reflecting from sample surfaces and ultrasonic wave velocity can be calculated assuming object or sample thickness is known. For example, [9] describes a method to determine welding stresses with an opto-piezoelectric technique that enables longitudinal wave TOF measurement with an accuracy of $0.5 \mathrm{~ns}$. However, to exploit this accuracy of TOF measurements and calculate velocity, sample thickness must be measured very precisely. To measure sample thickness with $2 \mu \mathrm{m}$ precision, the authors had to polish both sample surfaces. This example shows that velocity determination using the pulse-echo technique with longitudinal waves can be carried out under laboratory conditions only, with precisely machined samples.

Without precise information concerning element thickness, the only technique that can be applied in industrial conditions for ultrasonic stress evaluation seems to be a technique based on the measurement of acoustic birefringence. In such experiments, two TOF's of shear SH waves polarized parallel to the element surface are measured. The shear $\mathrm{SH}$ waves are polarized in orthogonal directions coinciding with principal stress directions. The relative TOF difference is proportional to the principal stress difference. From a practical point of view, an advantage of this technique is that detailed information about sample thickness is not necessary. Another advantage is that results of measurements are not temperature dependent, as it can be assumed that any temperature induced velocity changes are the same for both shear waves. These advantages of the acoustic birefringence technique led to wide application of evaluation of residual stress in railroad monoblock wheels. Two parallel rim faces allow the measurement of acoustic birefringence using a pulse-echo method and the calculation of hoop stress averaged between rim faces. Work [10] and reports [11, 12] describe the stress evaluation based on acoustic birefringence in monoblock wheels, using both electromagnetic-acoustic (EMAT) and piezoelectric transducers. Today, this technique of stress evaluation in forged monoblock wheels is a daily routine in many workshops in Europe.

\subsection{Method Using Subsurface or Surface Waves}

The second method to measure TOF and evaluate velocity is to use ultrasonic waves propagating parallel to the sample surface. For such measurements, access to only one flat or cylindrical surface of the sample is necessary and measurements can be performed on samples with complicated shapes. Railroad rail is a good example of such an object. Typically, the only place on the rail profile where two parallel surfaces can be found and the pulse-echo method can be applied is the center of the rail web. Subsurface waves can, however, be propagated along the rail at numerous places, such as the rail head running surface or sides, the rail web, or the upper and lower sides of the foot/base. In contrast to the pulse-echo technique, measurements with surface or subsurface waves allow the determination of stresses averaged in a thin material layer, thickness comparable to the wavelength, between the probes generating and receiving ultrasonic pulses.

If stress changes only are to be determined, a simple set of two probeheads can be used. Such a set, consisting of a transmitter and receiver, fixed to the surface of the sample under test, is shown in Fig. 1. To evaluate applied stress, the TOF measured before load application is treated as a "zero stress" TOF ( $t^{0}$ in formula (1)). It is assumed, as when using resistance strain gauges, that prior to the application of external forces, the material is unstressed or the stress state is known. An application of external loading results in ultrasonic wave TOF change due to two phenomena: the acoustoelastic effect that influences ultrasonic pulse velocity and a strain that changes the distance between probes fixed to the element surface. Depending on the mode of ultrasonic waves used in the measurement and material Young modulus, these two factors affect TOF to various degrees. For Rayleigh waves propagating parallel to stress in steel, about $75 \%$ of the total TOF change is the result of strain and the rest the result of the acoustoelastic effect. For the most 
Fig. 1 Schema of stress changes evaluation with two probes fixed to the element surface for surface or subsurface waves

Fig. 2 Set of transmitter-receiver probes fixed to the spacing bar
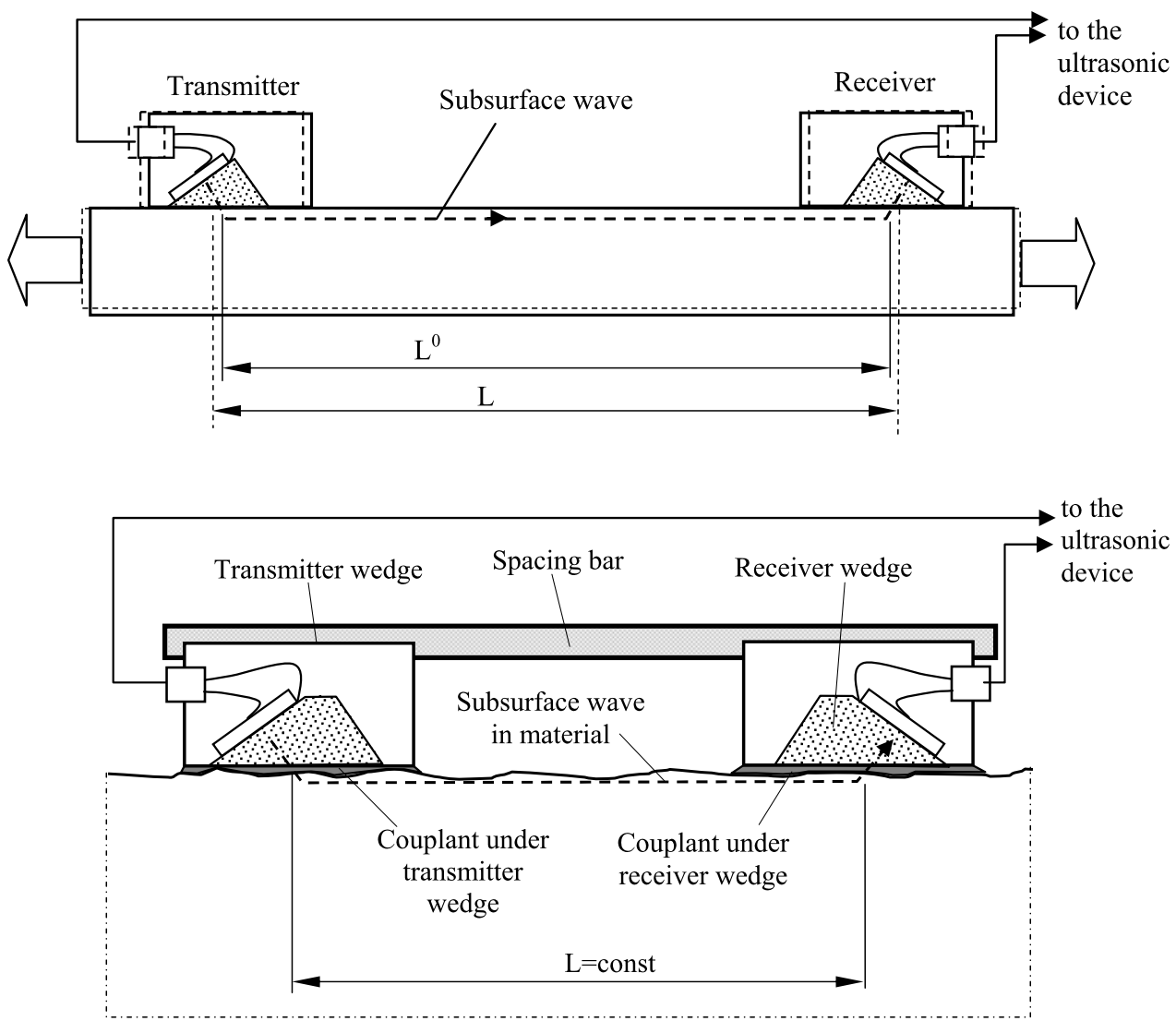

"stress sensitive" longitudinal waves propagating in steel, TOF changes are about $72 \%$ due to the acoustoelastic effect and only $28 \%$ the result of material deformation.

Monitoring stress changes with technique described above proved to be reliable method of stress evaluation. For example, stress changes in a girder of a steel bridge, performed with electromagnetic-acoustic (EMAT) transducers transmitting and receiving surface waves are described in [13]. Measurements of stress changes in a railroad bridge using both surface and longitudinal waves with piezoelectric transducers are presented in [14]. The application of Lamb waves for stress monitoring in thin walled composite tanks is described in [15]. In [16] a set of piezoelectric probes fixed with steel bolts to a railroad rail to monitor thermal stress changes in the rail is presented.

In measurements of stress change with sets of probes fixed to the element surface, the only factors influencing the measured TOF are stress, strain and temperature. The temperature influence on pulse velocity may occur in the sample under investigation and in probe wedges, which are usually made of plastic. Due to thermal expansion, the distance between probes also changes, as well as dimensions of the wedges. The combined influence of temperature on measured TOF can be determined experimentally in a temperature chamber on a sample made from the material investigated. Acoustic coupling between probes fixed to the sample surface is constant, neglecting temperature induced velocity changes in a relatively thin couplant liquid.

In the case of residual stress evaluation with surface or subsurface waves, zero stress TOF ( $t^{0}$ in Eq. (1)) and TOF in stressed condition ( $t$ in Eq. (1)) have to be measured on different samples. Zero stress TOF is measured on a stress relieved, annealed sample made of the same material as that under investigation. The TOF in stressed condition is measured on the object under test, quite often in numerous positions on the sample surface. Probes for such measurements have to be fastened to the spacing bar or plate that maintains constant distance between them as shown in Fig. 2. The constant distance between probes also results in the stress induced TOF difference between measurement on the stress free calibration sample and on the stressed element being lower compared to live stress monitoring with probes fixed to the surface-there is no influence of strain on TOF measured in the stressed state.

Acoustic coupling conditions can vary for measurements on various samples or in numerous positions along a single sample, depending on the surface condition variation. These coupling variations affect measured TOF, especially when the element surface is rough. When piezoelectric transducers are used, coupling variation seems to be the main reason for scatter in the TOF readings. To minimize coupling influence on the measured TOF of subsurface or surface waves, 
Fig. 3 Set of probes composed of one transmitter and two receivers (TRR set)

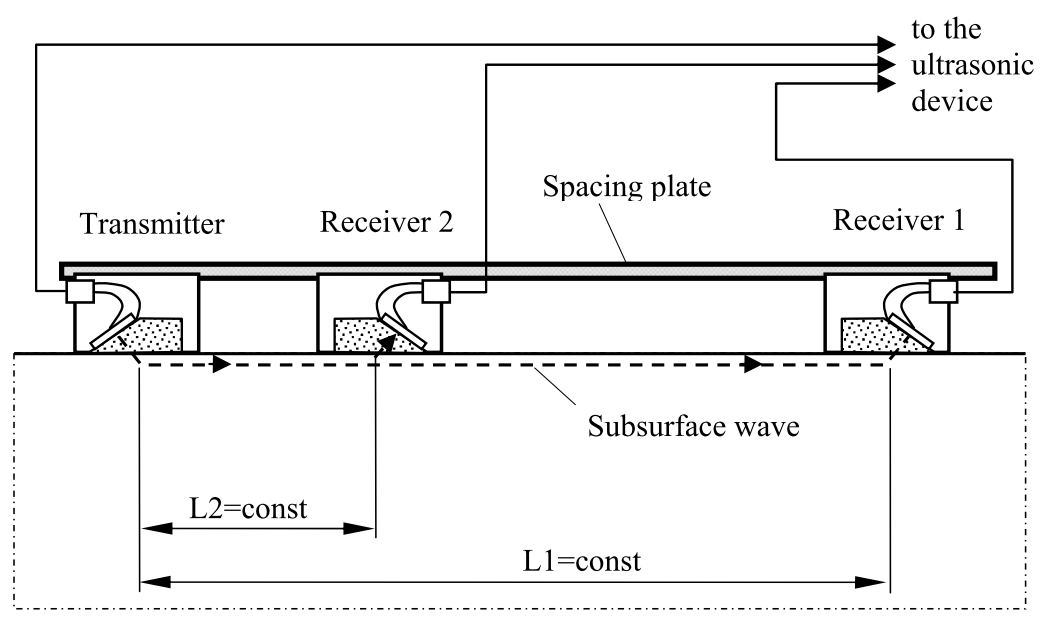

EMAT transducers, operating in contactless mode without liquid couplant, were tested. Experiments showed, however, that TOF measured with these transducers is sensitive to liftoff which depends on sample surface roughness [17]. Another important weak aspect of EMAT transducers is that their efficiency in generating and detecting longitudinal subsurface waves, whose acoustoelastic constant is the highest, is very poor. Therefore, in measurements using primarily longitudinal subsurface waves, using piezoelectric transducers is much more effective.

\subsection{Piezoelectric Probe Sets}

\subsubsection{Transmitter-Receiver Set}

Figure 2 shows a simple transmitter-receiver (TR) set of probes coupled to a rough surface with liquid couplant.

Subsurface wave TOF measured with TR set is equal to

$t=t_{W T}+t_{C T}+t_{M}+t_{C R}+t_{W R}$

where $t_{W T}$ - time of flight in transmitter wedge, $t_{C T}$ - time of flight in couplant under transmitting probe, $t_{M}$ - time of flight in material as a subsurface wave, $t_{C R}$ - time of flight in couplant under receiving probe, $t_{W R}$ - time of flight in receiver wedge.

Measurements with simple TR sets of probes are described in numerous papers. In [18] the authors studied the influence of surface roughness on TOF of surface waves and concluded that the sample surface should be ground smooth before stress evaluation with such a set of probes. Similar conclusions concerning surface roughness can be found in $[16,19]$.

In [20], describing a TR set of probes for Rayleigh waves, the authors noticed a significant temperature influence on TOF readings. They also found that for a given distance between probes in the set, the scatter of TOF readings depends on piezoelectric transducer size. For bigger transducers, the wave, depending on coupling, can propagate out of parallel to the axis of the probe set. A similar observation concerning transducer size was presented in [21] describing measurements with a TR set of EMAT transducers.

Despite the high sensitivity of TR sets of probes to coupling conditions, such probe sets were used to evaluate residual stresses in railroad wheels [22] and welds [23] in laboratory conditions at constant temperature. To achieve proper results, both wheels and weld had to be ground prior to taking measurements. In [23], the authors used a plastic bar to maintain the distance between transmitter and receiver. The high thermal expansion of such material increases the temperature influence on measurement results.

\subsubsection{Transmitter-Receiver-Receiver Sets}

Some advantages in precise TOF measurements are made by using a set of probes comprised of one transmitter and two receivers situated along the same line (TRR set). Such a set, called "double reception" or a "differential" set of probes, is shown in Fig. 3. In the case of piezoelectric transducers on plastic wedges, the temperature effect is reduced and to some degree, the influence of couplant variation on the readings is reduced.

The TRR set allows one to measure TOF as a difference between the TOF from the transmitter and the first receiver and the transmitter and the second receiver. This differential $t_{D}$ is equal:

$$
\begin{aligned}
t_{D}= & \left(t_{W T}+t_{C T}+t_{M 1}+t_{C R 1}+t_{W R 1}\right) \\
& -\left(t_{W T}+t_{C T}+t_{M 2}+t_{C R 2}+t_{W R 2}\right)
\end{aligned}
$$

where $t_{W T}$ - time of flight in transmitter wedge, $t_{C T}$ - time of flight in couplant under transmitting probe, $t_{M 1}, t_{M 2}-$ times of flight in the material as a subsurface wave between transmitter and receiver 1 and receiver 2 respectively, $t_{C R 1}$, $t_{C R 2}$ - times of flight in couplant under receiver 1 and 2 respectively, $t_{W R 1}, t_{W R 2}$ - times of flight in the wedge of receiver 1 and 2 respectively. 
Fig. 4 Design of three probe arrangement (TRR set) for surface roughness influence investigation on TOF of longitudinal $L$ and shear $S V$ waves
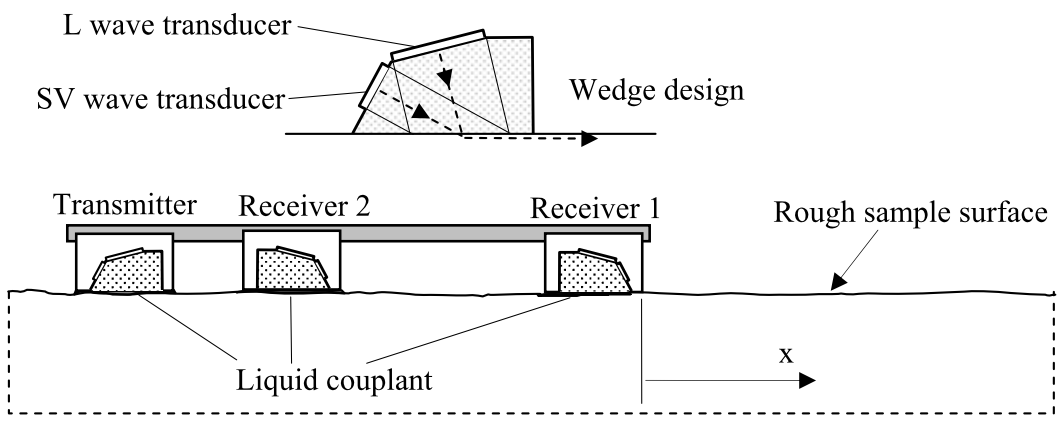

In theory, if the receiving wedges are identical, the differential TOF is equal to the time necessary for an ultrasonic pulse to travel in the material for the distance $L 1-L 2$. This compensation occurs, however, only when coupling conditions under both receivers are exactly the same $\left(t_{C R 2}=\right.$ $\left.t_{C R 1}\right)$. A second advantage of a TRR set as compared to a TR set is that, assuming both receiving probe wedges have the same geometry, the temperature influence on TOF in these wedges is also compensated.

TRR sets for longitudinal subsurface waves found application in residual stress evaluations in various samples. In [24] measurements on conventionally welded steel plates and on [25] laser welded plates are described. Paper [26] discusses the influence of sample bending on TOF measured with a TRR set of probes. Paper [8] presents the experiments with a TRR set for longitudinal subsurface waves having a time resolution of 0.2 ns. However, despite the compensating feature of TRR sets, use of such a high time resolution required the sample to be precisely machined before the measurements.

Samples in which residual stresses were evaluated using TR or TRR sets of piezoelectric probes, as described in the literature, all needed specially prepared surfaces. The aim of machining or grinding was to reduce the measured TOF scatter due to coupling variations. It is obvious that such preparations are impractical in industrial conditions. For practical applications, the ultrasonic evaluation of residual stresses should be nondestructive, fast and cheap. This means that measurements should be performed on the object without time consuming and expensive surface preparation like grinding or polishing. The following sections describe a set of piezoelectric probes that reduces the unwanted influence of surface roughness on readings to an acceptable level.

\section{Influence of Coupling Conditions on Subsurface Wave TOF}

In the ultrasonic testing of materials using angle beam probes a couplant, usually a liquid, grease or water-based gel is used. Longitudinal wave velocity in such media is about four times lower compared to its velocity in metal. Therefore, even small couplant thickness variations, related to surface roughness, result in relatively high TOF scatter.

Now, assume that the residual stress value has to be determined with an accuracy of $\pm 15 \mathrm{MPa}$. This accuracy of stress evaluation is equivalent, for longitudinal waves propagating over a distance of $150 \mathrm{~mm}$, to a TOF resolution of about plus/minus $5 \mathrm{~ns}$ (calculated for $\beta=-1.25 \cdot 10^{-5} \mathrm{MPa}^{-1}$ and velocity $v=5.94 \mathrm{~mm} / \mu \mathrm{s})$. In the case of liquid couplant, with a longitudinal wave velocity of about $1.5 \mathrm{~mm} / \mu \mathrm{s}$, $5 \mathrm{~ns}$ corresponds to a couplant thickness equal to only $0.0075 \mathrm{~mm}$. This means that to maintain $5 \mathrm{~ns}$ TOF repeatability, surface roughness should be controlled on a micron scale. Therefore, sets of probes described in the literature can be only used on specially prepared samples.

Figure 4 presents a set of three special probes to investigate the influence of surface roughness. On each of the plastic wedges, two piezoelectric transducers are positioned. One is used to produce a subsurface longitudinal wave, the second for subsurface shear $S V$ wave. The shape of the wedge ensures that ultrasonic beams produced by both transducers enter a steel sample at the same point, crossing an identical liquid couplant layer. Wedges of receiving probes were spaced by $115 \mathrm{~mm}$ and connected to a flexible brass plate to ensure constant distance between them. The set was equipped with magnets attracting them to sample surface, to ensure repeatable coupling conditions. Using such a set of probes for longitudinal and shear $S V$ waves, TOFs were measured for various positions on a rough steel sample. The sample was cut from a new rail head and its surface was only lightly cleaned with sandpaper to remove debris and loose scale. It can be assumed that over a distance of $40 \mathrm{~mm}$ along the rail, material texture and tensile residual stress are constant, and observed changes in TOF are due to surface roughness only. Figure 5 shows TOF measured in $2 \mathrm{~mm}$ increments along the sample. The TOF changes are related to TOF measured for both longitudinal and shear waves for $x=0 \mathrm{~mm}$. It can be seen that, despite different angles of incidence of ultrasonic waves on the sample surface, TOF changes resulting from varying couplant conditions are similar for both longitudinal and shear waves. Results show that after calibration on a flat, polished sample, shear wave 


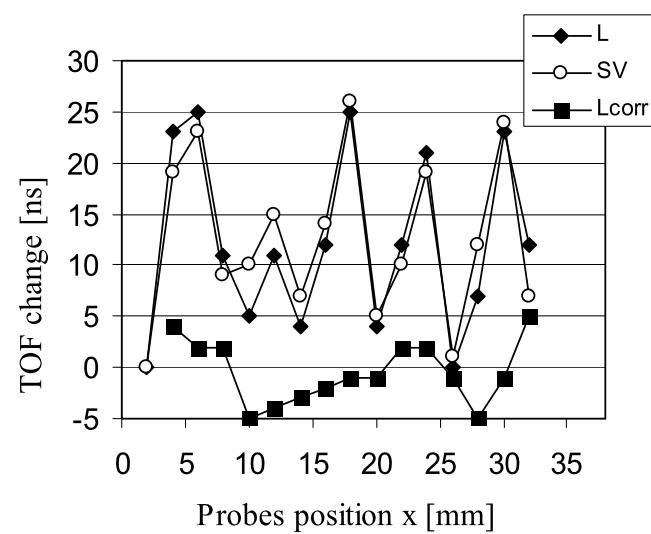

Fig. 5 Distribution of longitudinal $L$ and shear $S V$ subsurface waves TOF variations measured with three probe arrangement (TRR set) of double-transducer probes and corrected $L_{C o r r}$ longitudinal wave TOF

TOF can be used as a couplant variation indicator. A corrected longitudinal wave coupling $\left(t_{L_{C o r r}}\right)$ can be calculated as:

$t_{L_{C o r r}}=t_{L}+\left(t_{S V}-t_{S V_{C a l}}\right)$

where $t_{L}$-time of longitudinal wave in the sample under test, $t_{S V}, t_{S V_{C a l}}$-shear wave times in the sample under test and in the smooth calibration sample.

The corrected longitudinal wave TOF is shown in Fig. 5 as a black squares.

The acoustoelastic constant for shear $S V$ waves propagating parallel to stress in railroad rail steel is $\beta_{112}=$ $-0.08 \cdot 10^{-5} \mathrm{MPa}^{-1}$. This is about 15 times smaller than the acoustoelastic constant $\beta_{111}$ for longitudinal waves, so it can be assumed that shear wave TOF is only weakly influenced by stress. According to data presented in Fig. 5, application of a shear $S V$ wave as a coupling indicator can reduce longitudinal wave TOF scatter from $\pm 12.5 \mathrm{~ns}$ to $\pm 5 \mathrm{~ns}$.

The results shown in Fig. 5 indicate that special TRR set of probes only partly eliminates couplant thickness variations on TOF readings and is not a reliable instrument for TOF measurements on rough surfaces. Without any correction, longitudinal wave TOF scatter equal to $\pm 12.5 \mathrm{~ns}$ correspond to a calculated stress scatter equal to $\pm 50 \mathrm{MPa}$. The correction proposed above reduces this scatter to $\pm 20 \mathrm{MPa}$.

Experiments performed with the set of probes presented above also showed that in many elements such as beams, rails, plates or rods, the measured shear $S V$ wave TOF presents higher scatter compared to the longitudinal wave TOF. This is due to unavoidable transformations of shear waves on the sample into longitudinal waves, which travel about two times faster. Small amplitude signals after numerous transformations that reach the shear wave receiving transducer simultaneously with the main shear $S V$ pulse propagating parallel to the surface result in interference, confounding precise shear wave TOF readings.

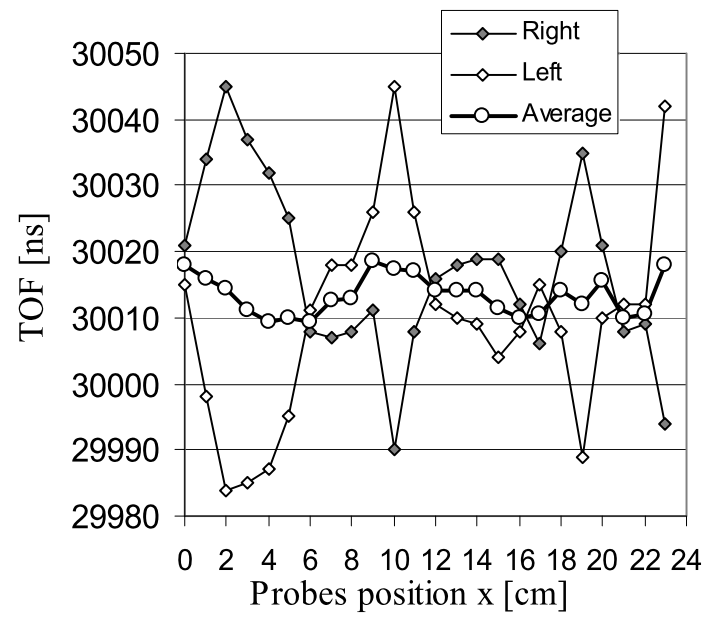

Fig. 6 Results of longitudinal subsurface wave TOF measurements with the set of three probes (TRR set) for longitudinal waves propagating in one direction (right) and in opposite direction (left) along the rough sample

\section{Multi-transducer Set of Probes}

\subsection{Probe Configurations}

During measurements performed with a TRR set of probes, it was observed that readings taken with longitudinal waves propagating in one direction and in opposite direction (after rotation of the set by $180^{\circ}$ ) in the same positions of receivers on the rough sample, indicate a mirror-like distribution. This effect can be explained as the influence of various couplant layers under receiving probes 1 and 2 (see Fig. 3). If there is a pit or a cavity on the sample surface located under receiver 1, time $t_{C R 1}$ (Eq. (3)) is longer. In result time $t_{D}$ is also longer. After rotation of the set, the pit is located under receiver 2 and time $t_{C R 2}$ is longer, what results in shorter time $t_{D}$.

Results of measurements performed with set of probes coupled twice, for waves propagating in both directions, taken along the new rail head running surface are shown in Fig. 6. Scatter in TOF for the wave propagating in one direction is $55 \mathrm{~ns}$ and in the opposite direction is about $61 \mathrm{~ns}$. The mean TOF value calculated for each position on the sample, shown with a open circles on the diagram, presents scatter of $\pm 5 \mathrm{~ns}$ only. This means that TOF averaging reduces scatter induced by roughness by a factor of 5. Consideration of the "mirror" effect led to the design of a 6-transducer set of probes (2T4R), shown in Fig. 7. Two transmitters, one generating the wave traveling "right", the second "left", are positioned along one line. Between them are situated two receiving probes. On the symmetrical wedges of each receiving probe are located two piezoelectric transducers. One of them detects pulses approaching from "right" and the second from the "left". Ultrasonic waves approaching from "right" and "left" enter the receiving wedge, 
Fig. 7 Six-transducer set of probes (2T4R set) for subsurface wave TOF measurements

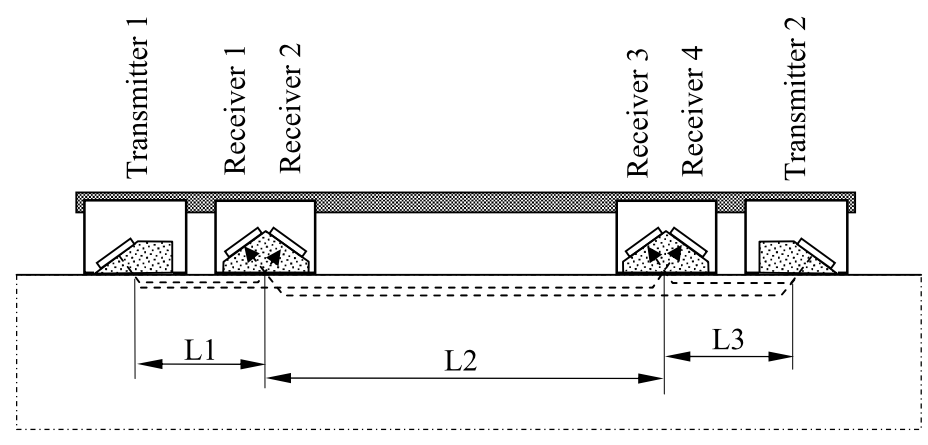

crossing the same couplant volume. Details of the shape of the wedges are presented in [27].

To calculate coupling compensated time $t_{\text {Comp }}$, four TOF measurements have to be performed:

1. $t^{1}$ between transmitter 1 and receiving transducer 2 ,

2. $t^{2}$ between transmitter 1 and receiving transducer 4 ,

3. $t^{3}$ between transmitter 2 and receiving transducer 3 ,

4. $t^{4}$ between transmitter 2 and receiving transducer 1 .

These TOF are equal:

1. $t^{1}=t_{W T 1}+t_{C W T 1}+t_{L 1}+t_{C W R 12}+t_{W R 2}$

2. $t^{2}=t_{W T 1}+t_{C W T 1}+t_{L 1}+t_{L 2}+t_{C W R 34}+t_{W R 4}$

3. $t^{3}=t_{W T 2}+t_{C W T 2}+t_{L 3}+t_{C W R 34}+t_{W R 3}$

4. $t^{4}=t_{W T 2}+t_{C W T 2}+t_{L 3}+t_{L 2}+t_{C W R 12}+t_{W R 1}$

where $t_{W T 1}, t_{W T 2}$-times in transmitter 1 and 2 wedges, respectively, $t_{C W T 1}, t_{C W T 2}$ - times in couplant under transmitters 1 and 2 , respectively, $t_{L 1}, t_{L 2}, t_{L 3}$-times in material under test, $t_{C W R 12}, t_{C W R 34}$ - times in couplant under receiving wedges with receivers $1-2$ and 3-4, respectively, $t_{W R 1}$, $t_{W R 2}, t_{W R 3}, t_{W R 4}$-times in receiving wedges to receiving transducers 1, 2, 3 and 4, respectively.

Differential time is calculated as:

$t_{D}=\left(t^{2}-t^{1}+t^{4}-t^{3}\right) / 2=t_{L 2}$

Assuming that the wedges of the receiving probes are symmetrical $\left(t_{W R 2}=t_{W R 1}\right.$ and $\left.t_{W R 3}=t_{W R 4}\right)$, the differential time $t_{D}$ is compensated for couplant thickness variations under receiving probes.

It was also observed that for small transmitting transducers situated away from the receivers (distances $L 1$ and $L 2$ on Fig. 7), coupling conditions under transmitting wedges do not influence the measured TOF.

Figure 8 shows a comparison of subsurface longitudinal wave TOF measured in numerous locations along the rail head using various sets of probes. The distance between receivers for all sets was the same, $198 \mathrm{~mm}$, and the measurements were taken every $10 \mathrm{~mm}$. According to expectations, the highest TOF scatter observed was for the TR set. Use of a TRR set results in about $50 \%$ lower TOF scatter. Scatter

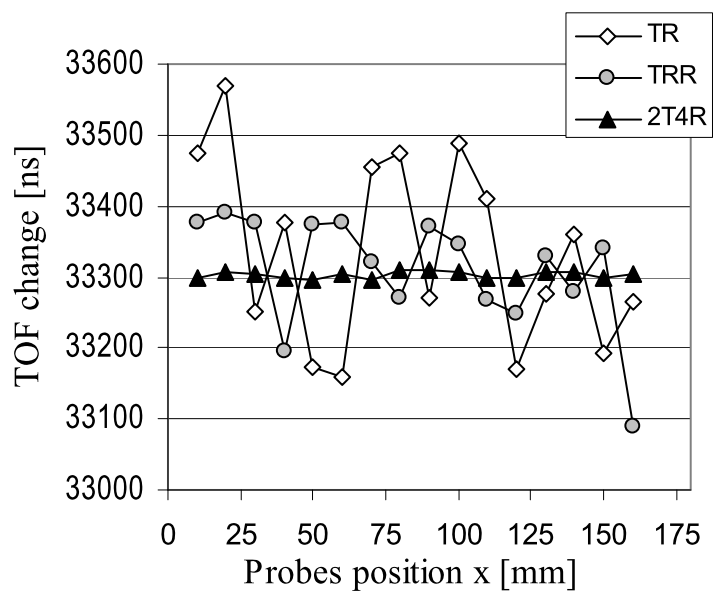

Fig. 8 TOF of subsurface longitudinal wave measured with various sets of probes (TR, TRR and 2T4R) along rail head

for a 2T4R set was significantly lower and equal to only few nanoseconds.

Ratios of maximum and minimum TOF values measured with three set of probes on the rail, as compared to mean TOF value were:

$+0.7 \%, \quad-0.5 \%$ for TR set,

$+0.24 \%, \quad-0.66 \%$ for TRR set,

$+0.02 \%, \quad-0.02 \%$ for 2T4R set.

The 2T4R set described above was developed for residual stress evaluation in railroad rails. Experience during numerous experiments on various rails showed that, in practice, reliable measurements in industrial conditions, at numerous locations along the rail, are possible only with 6-transducers sets. Obtaining the same amount of data with a TRR set would require time consuming multiple measurements and TOF averaging or impractical surface preparation in order to minimize roughness influence on readings.

It must be mentioned however that $2 \mathrm{~T} 4 \mathrm{R}$ set of probes, equipped with small transducers, eliminates unwanted coupling influence on readings only for limited roughness height. Measurements on very rough, corroded steel sam- 
Fig. 9 Angular distribution of acoustic pressure in critically refracted longitudinal wave beam, for various transducer sizes

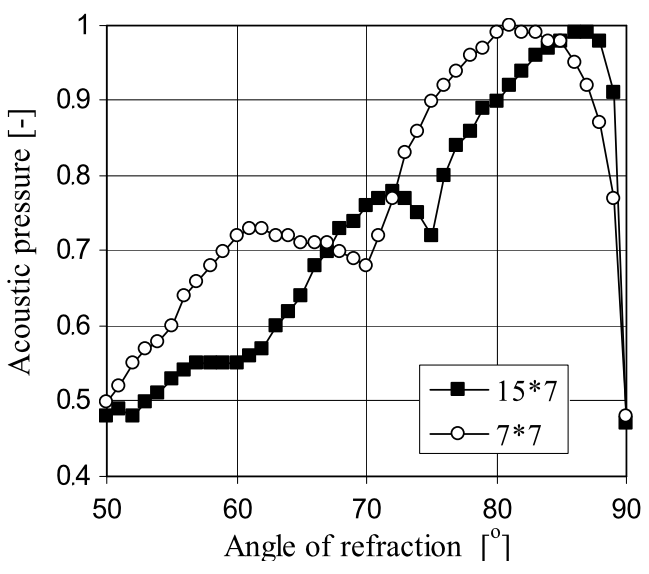

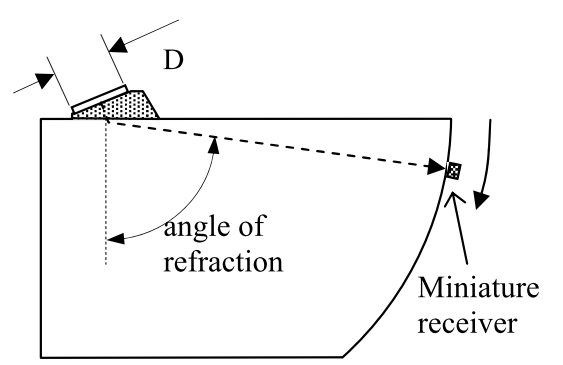

ples (maximum roughness height $0.3-0.5 \mathrm{~mm}$ ) showed unaccepted TOF scatter equal to about $\pm 50 \mathrm{~ns}$.

\subsection{T4R Set Design}

To generate a critically refracted longitudinal (subsurface) wave with maximum acoustic pressure close to the sample surface, large dimension transducers should be used. Experiments confirm that a higher refraction angle is observed for larger transducer size/wavelength ratio. Figure 9 shows the angular distribution of acoustic pressure for transducers with height of 15 and $7 \mathrm{~mm}$ (by $7 \mathrm{~mm}$ wide), as experimentally determined in a steel sample. A longitudinal wave was detected and its amplitude evaluated using a small receiving transducer coupled to the cylindrical surface of the sample.

It can be seen that maximum pressure radius for the smaller transducer is observed at a lower angle of refraction than for larger one, in agreement with theoretical expectations. However, a larger transducer necessitates a larger plastic wedge. The increased height of a larger wedge means a longer TOF of the pulse in a wedge material, with higher influence of any temperature gradients in the wedge or temperature difference between wedges on measured TOF. Therefore, in probes designed for residual stress evaluation in rails and monoblock wheels, to minimize an unwanted temperature influence on TOF, small $(7 \times 7 \mathrm{~mm})$ transducers, positioned on small wedges were used. Experiments showed that $4 \mathrm{MHz}$ transducers, driven by standard ultrasonic pulser, ensure acceptable pulse amplitudes for measurements in steel on distances between transmitter and receiver up to $200 \mathrm{~mm}$.

An additional factor influencing TOF measured for subsurface waves is elasticity of a spacing bar connecting the probes. It was noticed that on rough surfaces, a lower TOF scatter was measured with probes connected with a spacing bar made of flexible metal plate. To ensure constant coupling force, each of the probes in the set was equipped with a strong permanent magnet. To reduce the wear of relatively soft plastic wedges in contact with rough steel surfaces, each plastic wedge was also located in a hard steel housing.

\subsection{Temperature Correction}

Temperature significantly influences the results of subsurface wave TOF measurements. Velocities of ultrasonic waves in the material under test and in plastic wedges depend on temperature. For steel, the temperature induced velocity of a longitudinal wave change is about $-0.55 \mathrm{~m} / \mathrm{s}$ per degree Celsius. A much higher velocity-temperature dependence and thermal expansion is observed for plastics (PMM, Rexolite) used for wedges. For a wedge made of PMM, the influence of temperature on the velocity of longitudinal waves is about $-2.5 \mathrm{~m} / \mathrm{s}$ per degree Celsius. For example, a $10{ }^{\circ} \mathrm{C}$ temperature increase results in a $50 \mathrm{~ns}$ increase of longitudinal wave TOF in $7 \mathrm{~mm}$ tall PMM wedge, due to velocity change and thermal expansion. Even small temperature differences between receiver wedges can lead to significant changes of measured TOF.

Another factor that influences TOF measured with sets of probes for subsurface waves is the thermal expansion of the spacing bar or plate. Elongation of this bar increases the distance between probes. In the final 2T4R set design to minimize thermal elongation of the plate, probes were connected with a flexible plate made of INVAR. The thermal expansion coefficient for INVAR, equal to $1.5 \cdot 10^{-6}\left[{ }^{\circ} \mathrm{C}^{-1}\right]$ is twelve times lower comparing to coefficient of brass, equal to $18.7 \cdot 10^{-6}\left[{ }^{\circ} \mathrm{C}^{-1}\right]$. The temperature also influences the capacitance of piezoelectric transducers and therefore the resulting frequency of ultrasonic pulses. It influences also the angle of refraction at the wedge/couplant/steel interface. But in the temperature range in which subsurface waves can be generated and detected with piezoelectric probes these influences, as compared to other mentioned factors, can be neglected.

The only practical and effective way to determine the temperature influence on TOF measured using a specific set of probes is experimental evaluation of TOF-temperature dependence. This can be performed in a temperature chamber with a set of probes coupled to the smooth sample made 


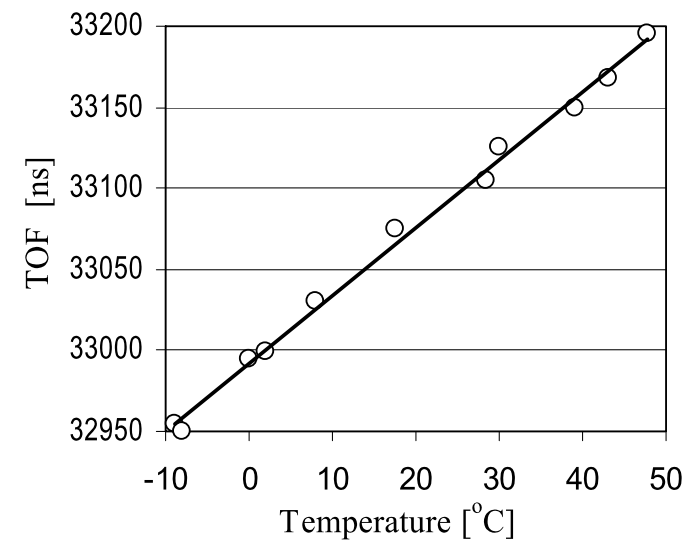

Fig. 10 Temperature induced TOF changes measured with a six transducer set (2T4R) of probes equipped with brass spacing plate

of the material to be tested using the liquid couplant. Figure 10 shows an example of such a measurement performed with a 2T4R set coupled to a steel sample. The distance between receivers was $196 \mathrm{~mm}$ and probes were connected to a flexible plate made of brass. It can be seen that $10{ }^{\circ} \mathrm{C}$ temperature increase results in about $35 \mathrm{~ns}$ TOF increase. Temperature correction for this set is equal to $-3.5 \mathrm{~ns} /{ }^{\circ} \mathrm{C}$. The observed TOF shift is mostly due to thermal expansion of brass plate. Application of INVAR reduces the temperature induced TOF shift by a factor of 5 .

Sets of probes designed for field measurements were equipped with temperature sensors located close to the element surface. The TOF correction for temperature corrected was calculated as:

$t_{T C}=t_{T}+C_{T}\left(T_{r e f}-T\right)$

where $C_{T}$-temperature correction determined experimentally, $T_{r e f}, T$-reference and measured temperatures, respectively, $t_{T}$-time of flight measured in temperature $T$.

Quite often in measurements in industrial conditions, the temperatures of the probe set and object to be measured differ. To minimize the time necessary for the wedges and spacing bar to reach the same temperature as a sample under test, is it advisable to minimize heat capacity of the set and maximize heat flow from the sample to the spacing plate. Also, probes and the connecting spacing bar should be thermally isolated from any other elements of the set, such as electronic circuits and housing. Liquid couplant and boxes surrounding and protecting the plastic wedges against wear help to transport heat from the object to the wedges and spacing plate. Nevertheless, the time necessary to equalize temperature in the case of a cold set of probes coupled to the warm test objects can be significant. Figure 11 presents the TOF measured with a $2 \mathrm{~T} 4 \mathrm{R}$ set of probes at room temperature coupled to a head running surface of a cold $\left(-5^{\circ} \mathrm{C}\right)$ $0.5 \mathrm{~m}$ long section of UIC60 rail. The temperature indicated by a sensor mounted in the probe set close to the rail surface

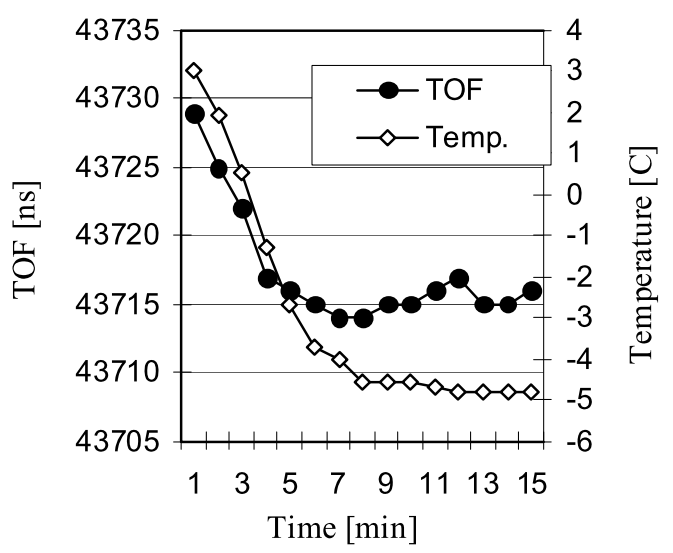

Fig. 11 Changes of TOF measured with six transducer set of probes (2T4R set) coupled to the head running surface of cold UIC60 rail section. Initial set and sample temperatures: $23^{\circ} \mathrm{C}$ and $-5^{\circ} \mathrm{C}$ respectively

is shown on the diagram. Readings taken every minute show that measured TOF stabilizes after a significant time. For this set of probes, the time necessary to equalize temperatures was about 8 minutes. During this time period, the measured TOF drops down by $15 \mathrm{~ns}$ for a given set of probes, which corresponds to a stress change equal to $27 \mathrm{MPa}$. It is likely that when using sets of probes equipped with larger transducers positioned on taller plastic wedges, without any metal frames surrounding them and supporting heat flow, the time necessary for TOF reading stabilization would be much longer.

\section{Examples of Application of Multi-transducer Set of Probes}

The 2T4R sets of probes described above were used mostly for residual stress evaluation in the railroad industry. Different distances between receiving probes and different frequencies of ultrasonic wave were chosen depending on the application. For the evaluation of the longitudinal component of residual stress in railroad rails after straightening, the distance between receivers and test frequency were $193 \mathrm{~mm}$ and $4 \mathrm{MHz}$, respectively.

Another application was the evaluation of longitudinal stress (called also thermal stress) in continuously welded rail. For this application, due to lower absolute stress values, the distance between receiving probes was longer, about $300 \mathrm{~mm}$. To obtain an acceptable pulse amplitude, it was necessary to use larger transducers and to reduce the test frequency to $2 \mathrm{MHz}$. Measurements were to be performed at various temperatures, so the wedge angle was selected to provide an acceptable pulse amplitude for higher rail temperatures. The length of the set designed to evaluate hoop component of residual stress in the rim of monoblock wheel 


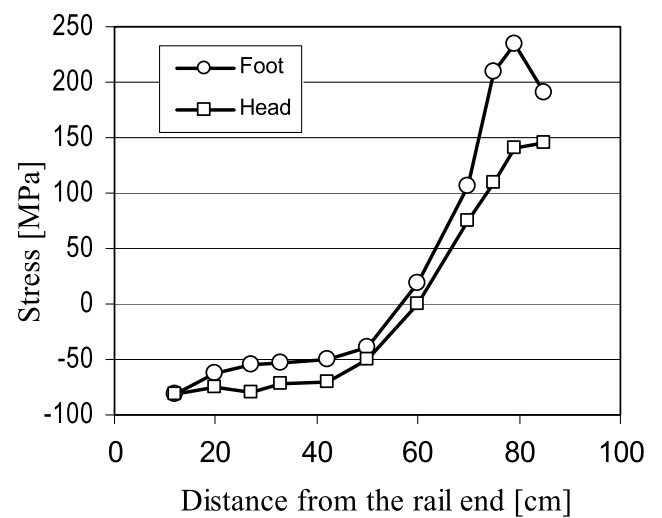

Fig. 12 Longitudinal component of residual stress in the rail after straightening. Stress distribution in the head and foot, along the leading end of the rail

was limited by the width of the flat rim face. Therefore, the distance between receivers was about $160 \mathrm{~mm}$.

Figure 12 shows results of residual stress evaluation along the leading end of a rail after roller straightening. Before measurement, the device was calibrated (measurement of $t^{0}$ according to formula (1) for head and foot/base) on the rail section subjected to stress relieving annealing. Readings shown in Fig. 12 were taken on the rail head running surface and on the rail foot/base underside. Each stress value was calculated as averaged for three set positions spaced by 1-2 mm. Smooth distribution of stress value along the rail can be observed for both rail head and foot. The beginning of the rail exhibits stresses that resulted from rail heat treatment. Stresses along a distance of $80 \mathrm{~cm}$ from the rail are the result of plastic deformation during straightening.

Figure 13 shows the distribution of longitudinal stress (called also thermal stress) along a continuously welded rail (CWR) [28]. Readings were taken with a 2T4R set probes coupled to the side of the rail head. This part of the rail profile is not deformed by wheels of passing trains. It is also flat what allows to use set of probes equipped with big transducers, able to transmit and detect subsurface wave on the longer distance. It was assumed that at this spot on the rail profile, residual stresses introduced during rail manufacture are fairly constant and the only factors influencing TOF are temperature and longitudinal force in the CWR. The device was calibrated in each location before the rails were welded into CWR and were free of any external loads. Than readings were taken in the same locations after rails welding into CWR and track geometry adjustments, in the same temperature as calibration. Results of this measurements show longitudinal stresses introduced into the rail during track laying and geometry adjustment only. Second measurements were performed after 10 months of track operation again for the same rail temperature. These readings show changes in longitudinal force in the rail resulting from the traffic loads. After 10 month of service, the rail surface was corroded but

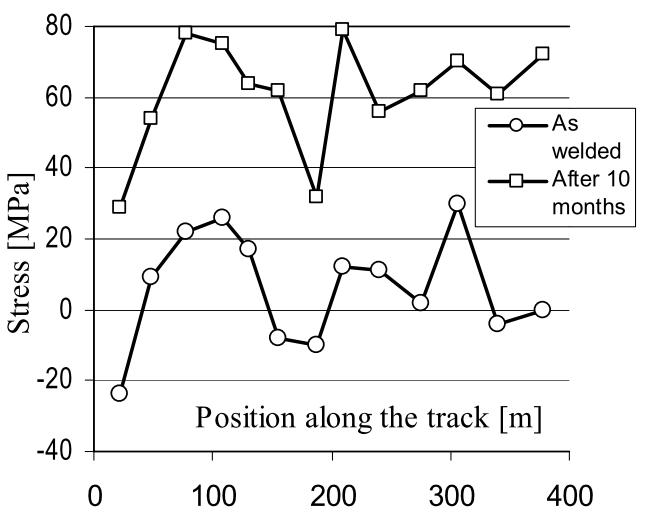

Fig. 13 Longitudinal stress (called also thermal stress) values in the continuously welded rail as welded (new track) and after 10 months of service

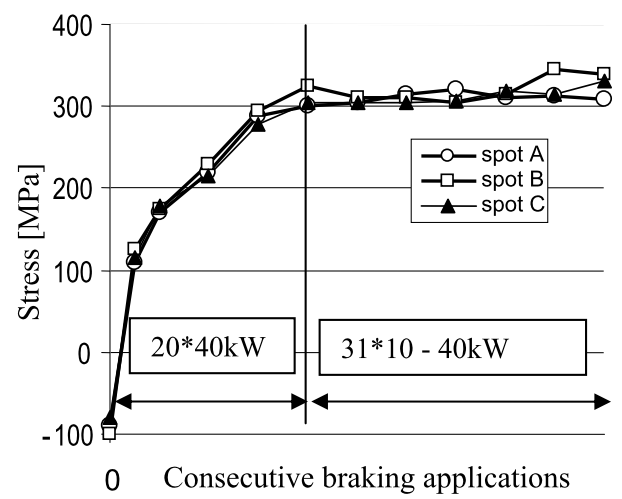

Fig. 14 Tensile hoop stress development in the monoblock wheel subjected to braking in the stand. Measurements with six transducer set (2T4R set) for subsurface longitudinal wave in three locations on front rim face

could still be evaluated with the 2T4R set of probes, and the stress distribution along the rail is similar to initial stresses. Note the relatively low stress values as compared to residual stresses evaluated in the rails after straightening from Fig. 12.

Figure 14 shows the results of measurements performed on railroad monoblock wheel subjected to braking in a test stand. TOF was measured on front rim face using a longitudinal subsurface wave propagating in the hoop direction (along the chord), at three locations on the wheel circumference. The device was calibrated on a portion of wheel rim that had been stress relieved. Hoop stress on the front rim face in the as manufactured state (before braking) was about $-100 \mathrm{MPa}$ (compression). The wheel was subjected to total 51 braking cycles at 60 minutes each. The first $40 \mathrm{~kW}$ power braking cycle changed the hoop stress from compressive to tensile. Further brake applications with high power increased the tensile stress to about $300 \mathrm{MPa}$. Last brakings, with power between 10 and $40 \mathrm{~kW}$, resulted in smaller tensile stress increases. 


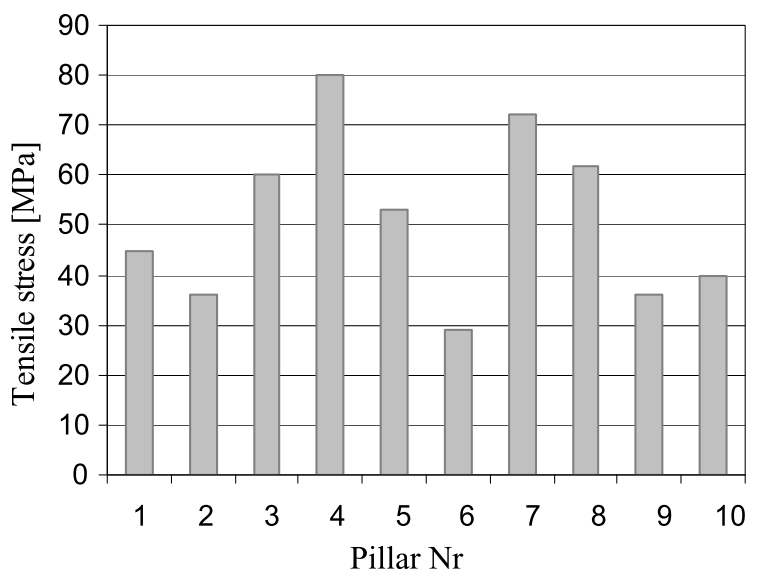

Fig. 15 Tensile stresses in 10 pillars of hydraulic press evaluated with six transducer set of probes (2T4R set) for longitudinal subsurface waves

The set of probes designed for thermal stress monitoring in rail in track was used to evaluate stress values in pillars of a nominal 20,000 ton hydraulic press in a fiberboard factory. Ten pillars, $350 \mathrm{~mm}$ in diameter and $6 \mathrm{~m}$ long, were subjected to tensile stresses during each pressing cycle. Stress value in an individual pillar depends on the distribution of fiber layers between upper and lower press frames. Stress depends also on pillars length differences and deformation of upper and lower frames during pressing. Fibers entering the press contain significant amount of liquid which pour press elements during initial period of press operation. The application of resistance strain gauges to monitor stress changes in pillars in such a conditions was very difficult and required complicated protection of the delicate gauges from heat and aggressive liquids that occasionally leaked on to the press pillars. Therefore, the only technique likely able to deliver reliable information regarding stress values in the pillars under these conditions was an ultrasonic method. Measurements were performed with longitudinal wave propagating along the pillar axis. Each pillar was measured at three positions spaced $120^{\circ}$ apart on its circumference. The aim of reading at various positions along the circumference was to verify if the pillars were also subjected to bending.

The first readings were taken at zero force. Longitudinal stress in this state was assumed to be zero, and mass forces and assembly stresses were neglected. The second readings were taken in the same positions during press operation, after a few minutes of pressing when the majority of liquid from the fiber charge had moved. Mean values of longitudinal stress in 10 pillars are presented in Fig. 15. Significant stress differences between individual pillars can be noticed. Maximum stress value was $80 \mathrm{MPa}$, while minimum was $29 \mathrm{MPa}$. Uneven cyclic loading of press pillars was the likely reason for fatigue cracks occurring in the pillars.

The acoustoelastic constant for steel in the pillars was unknown. For approximate stress calculations, it was assumed to be $\beta_{111}=-1.25 \cdot 10^{-5} \mathrm{MPa}^{-1}$. The mean value of stress in the pillars is equal to $51.3 \mathrm{MPa}$ what results in averaged tensile force during measurement of about 500 ton per pillar.

\section{Conclusions}

The literature describes various designs of TR and TRR sets of piezoelectric probes for stress evaluation with subsurface or surface waves, which can be used on prepared samples under laboratory conditions. Described above 2T4R sets of probes was developed to reduce the unwanted influence of coupling variations and temperature on TOF readings and to enable ultrasonic stress evaluation in industrial conditions. Special design probes of this general description were developed for specific applications. These applications included the evaluation of the longitudinal component of residual stress in the railroad rail after straightening, monitoring of longitudinal force (called also "thermal stress") changes in continuously welded rail and the evaluation of hoop residual stress on the faces of monoblock railroad wheel rims.

All measurements presented in Sect. 5 were performed under industrial conditions, on very large and heavy objects, in numerous locations of the probes on the objects. Measurements had to be done after cleaning and only hand sanding of the surface. For example, in the measurement of stresses in hydraulic press pillars, one 2T4R set of probes replaced 60 resistance strain gauges, assuming these could have been glued to the pillars. As compared to simple TR or TRR sets, six-transducer sets of probes increase the number of individual TOF measurements. Due to differences in ultrasonic pulse paths, consecutive pulses had various delays and amplitudes. All probe sets described in Sect. 5 were equipped with temperature sensors and temperature readings were necessary to calculate temperature corrected TOF. Therefore, to facilitate the measurements, 2T4R sets should be connected to a specially designed device that supports them. All measurements presented in this paper were performed with various DEBRO devices.

Open Access This article is distributed under the terms of the Creative Commons Attribution License which permits any use, distribution, and reproduction in any medium, provided the original author(s) and the source are credited.

\section{References}

1. Hughes, D.A., Kelly, G.S.: Second-order elastic deformation of solids. Phys. Rev. 92, 1145-1156 (1953)

2. Bergman, R.M., Shahbender, R.A.: Effect of statically applied stresses on the velocity of propagation of ultrasonic waves. J. Appl. Phys. 29, 1736-1738 (1958)

3. Benson, R.W.: Development of nondestructive methods for determining residual stress and fatigue damage in metals. NASA Marshall Space Flight Report, Contract No. NAS8-20208 Report (1968) 
4. Chaki, S., Bourse, G.: Stress level measurement in prestressed steel strands using acoustoelastic effect. Exp. Mech. 49, 673-681 (2009)

5. Clark, A.V., Fukuoka, H., Mitrakovic, D.V., Moulder, J.C.: Characterization of residual stress and texture in cast steel railroad wheels. Nondestruct. Test. Eval. 28, 119-126 (1995)

6. Deputat, J., Kwaszczynska-Klimek, A., Szelazek, J.: Monitoring of residual stress in railroad wheels with ultrasound. In: Proc. 12 WCNDT, vol. 2, pp. 974-976. Amsterdam, 1989 (1989).

7. Hasegawa, M.M., Matsumura, J., Kusano, R., Tsushima, S., Sasaki, Y., Oda, K.: Acoustoelastic effect for nondestructive stress measurement. Constr. Build. Mater. 24, 1713-1717 (2010)

8. Qozam, H., Chaki, S., Bourse, G., Robin, C., Walaszek, H., Bouteille, P.: Microstructure effect on the Lcr elastic wave for welding residual stress measurement. Exp. Mech. 50, 179-185 (2010)

9. Karabutov, A., Devichensky, A., Ivochkin, A., Lyamshev, M., Pelivanov, I., Rohadgi, U., Solomatin, V., Subudhi, M.: Laser ultrasonic diagnostics of residual stress. Ultrasonics 48, 631-635 (2008)

10. Schneider, E., Herzer, R., Bruche, D., Frotscher, H.: Ultrasonic characterization of stress states in rims of railroad wheels. In: Green, R.E. Jr., et al. (eds.) Nondestructive Characterization of Materials VI, pp. 383-390. Plenum, New York (1994)

11. Schramm, R.E., Szelążek, J., Clark, A.V.: Residual stress in induction heated railroad wheels: ultrasonic and saw cut measurements. NISTIR 5038, Report No. 28, National Institute of Standards and Technology, Boulder, CO (1995)

12. Schramm, R.E., Szelążek, J., Clark, A.V.: Dynamometer-induced residual stress in railroad wheels: ultrasonic and saw cut measurements. NISTIR 5043, Report No. 30, National Institute of Standards and Technology, Boulder, CO (1996)

13. Fuchs, P., Clark, A., Lozev, A.V., Halabe, U., Klinkhachorn, P., Petro, S., Ganga Rao, H.: Ultrasonic instrumentation for measuring applied stress on bridges. J. Nondestruct. Eval. 17, 141-152 (1998)

14. Szelążek, J., Gutkiewicz, P., Mackiewicz, S.: Dynamic stress monitoring with ultrasonic technique. In: Proc. SMART'09 Conf., 1315 July, Porto. Portugal, pp. 349-350 (2009)

15. Honhjian, H., Qing, H., Yong, Y.: A new nondestructive technique for measuring pressure in vessels by surface waves. Appl. Acoust. 69, 891-900 (2008)
16. Vangi, D., Virga, A.: A practical application of ultrasonic thermal stress monitoring in continuous welded rail. Exp. Mech. 47, 617623 (2007)

17. Morrison, J.P., Dixon, S., Potter, M.D., Jian, X.: Lift-off compensation for improved accuracy in ultrasonic lamb wave velocity measurements using electromagnetic acoustic transducers (EMATs). Ultrasonics 44, e1401-e1404 (2006). Supplement

18. Eryi, H., Yuming, H., Yanming, C.: Experimental study on the surface stress measurement with Rayleigh wave detection technique. Appl. Acoust. 70, 356-360 (2009)

19. Guz, A.N.: On foundations of the ultrasonic nondestructive method for determination of stresses in near-surface layers of solid bodies. Int. Appl. Mech. 41, 944-955 (2005)

20. Duquennoy, M., Ouaftouh, M., Ourak, M.: Ultrasonic evaluation of stresses in orthotropic materials using Rayleigh waves. Nondestruct. Test. Eval. 32, 189-199 (1999)

21. Murayama, R.: Non-destructive evaluation of formability in cold rolled steel sheets using the SH0-mode plate wave by electromagnetic acoustic transducer. Ultrasonics 39, 335-343 (2001)

22. Santos, A.A., Santos, G.F.M., Santos, F.C., Andrino, M.H.: Application of Lcr waves to evaluate residual stresses in the rim of railroad forged wheels. J. Nondestruct. Eval. 28, 91-100 (2009)

23. Tanala, E., Bourse, G., Fremiot, M., De Belleval, J.F.: Determination of near surface residual stresses on welded joints using ultrasonic methods. Nondestruct. Test. Eval. 28, 83-88 (1995)

24. Bray, D.E., Junghans, P.: Application of the LCR ultrasonic technique for evaluation of post-weld heat treatment in steel plates. Nondestruct. Test. Eval. 28, 235-242 (1995)

25. Ya, M., Marquette, P., Belahcene, F., Lu, J.: Residual stresses in laser welded aluminium plate by use of ultrasonic and optical methods. Mater. Sci. Eng. A 382, 257-264 (2004)

26. Bray, D.E., Tang, W.: Subsurface stress evaluation in steel plates and bars using the LCR ultrasonic wave. Nucl. Eng. Des. 207, 231-240 (2001)

27. Brokowski, A., Szelążek, J.: Set of ultrasonic probeheads for the measurement of time of flight of ultrasonic pulses. US Patent 5,549,001 (1996)

28. Szelążek, J.: Ultrasonic measurements of thermal stresses in continuously welded rails. Nondestruct. Test. Eval. 25, 77-85 (1992) 